\title{
Surface properties and scaling behavior of a generalized ballistic deposition model in $(1+1)$-dimension
}

\author{
Baisakhi Mal, ${ }^{1,2, \circledast ~ S u b h a n k a r ~ R a y, ~}$, , and J. Shamanna ${ }^{3, \text {, }}$ \\ ${ }^{1}$ Department of Physics, Jadavpur University, Calcutta 700 032, India. \\ ${ }^{2}$ Department of Physics, Budge Budge Institute of Technology, Calcutta 700 137, India. \\ ${ }^{3}$ Physics Department, University of Calcutta, Calcutta 700 009, India.
}

(Dated: July 2, 2018)

\begin{abstract}
The surface exponents, the scaling behavior and the bulk porosity of a generalized ballistic deposition (GBD) model are studied. In nature, there exist particles with varying degrees of stickiness ranging from completely non-sticky to fully sticky. Such particles may adhere to any one of the successively encountered surfaces, depending on a sticking probability that is governed by the underlying stochastic mechanism. The microscopic configurations possible in this model are much larger than those allowed in existing models of ballistic deposition and competitive growth models that seek to mix ballistic and random deposition processes. In this article, we find the scaling exponents for surface width and porosity for the proposed GBD model. In terms of scaled width $\widetilde{W}$ and scaled time $\tilde{t}$, the numerical data collapse on to a single curve, demonstrating successful scaling with sticking probability $p$ and system size $L$. Similar scaling behavior is also found for the porosity.
\end{abstract}

\section{INTRODUCTION}

The formation and growth of rough surfaces have several applications in physical and chemical processes, such as crystal growth, growth of thin films, vapor deposition, formations of colloids and electroplating to name a few [1, 2]. Many of the unique mechanical, optical and electromagnetic properties of surfaces originate from their surface morphology. The bulk properties of depository rocks, e.g., porosity, saline saturation, texture, stability and strength, find important applications in geology of sedimentary rocks. The underlying formation mechanism influences the geometry of deposition structures and is relevant in the manufacture of optical and electronic nanostructures and nanodevices, sophisticated drug delivery systems using magnetic carbon nanostructures [3] and smart nanostructures for monitoring, diagnoses and treatment in physiology [4]. Understanding the dynamics and growth of surfaces is, therefore, a challenging problem in surface science.

There are two fundamental approaches to the study of surface growth: (i) extensive numerical simulation of discrete models and computation of surface and bulk properties [2]; and (ii) solving the stochastic differential equation derived from phenomenology corresponding to the growth model [5, 6]. Another recent approach of study involves transformation of discrete deposition rules into stochastic differential equations using a limiting procedure and regularization, and hence, finding the scaling exponents [7].

Random deposition (RD) is a simple deposition process where non-sticky, solid particles deposit on randomly selected sites of a substrate (see Fig 1a). A quantitative

\footnotetext{
* baisakhi.mal@gmail.com

$\dagger$ sray.ju@gmail.com, sray@phys.jdvu.ac.in

$\ddagger$ jlsphy@caluniv.ac.in
}

measure of the roughness of the surface, called the surface width $W(L, t)$, is defined in terms of the surface height $h(i, t)$, at a site $i$ and at a time $t$, as,

$$
W(L, t)=\sqrt{\frac{1}{L} \sum_{i=1}^{L}[h(i, t)-\langle h(t)\rangle]^{2}},
$$

where $L$ is the system size, and $\langle>$ is the average. In RD, each site grows independently of the other sites and the surface roughness grows without bound. There are no voids in random deposition, so the deposition structure is compact [1].

Ballistic deposition (BD) on the other hand, gives rise to porous structures as the depositing particles stick to the first surface they encounter in their vertical downward journey towards randomly selected sites (see Fig 1b). In BD, the particles behave as strongly sticky, whereas in RD they are not sticky at all [1, 8, 9]. In nature however, particles may have intermediate stickiness which varies between the two extremes of strongly sticky and completely non-sticky behavior.

In the present study of a generalized ballistic deposition (GBD) model, we investigate the deposition of physically realistic particles with intermediate stickiness. The level of stickiness is parametrized in terms of a sticking probability $p$ of the incident particle, at each contact point with the surface. The parameter $p$ ranges between 0 and 1, 0 representing non-sticky particles as in the case of random deposition, and 1 representing extremely sticky particles, as in ballistic deposition. A logarithmic plot of the surface width $W(L, t)$ versus $t$, for GBD shows three distinct growth regions, similar to those observed by Banerjee et. al.[10], followed by saturation. The present model, for any $p>0$, however small, leads to porous structures [11]. Interesting scaling relations of surface roughness and porosity, with both the system size $L$, and the sticking probability $p$, are observed in the growth [6] and saturation regions. The relevant scaling 
exponents are determined from the simulation data.

This GBD is distinct from the earlier competitive growth models [12 22] in several ways. The deposition in GBD mimics a realistic sticking process such as gel, or mud thrown on a wall. Some variants of BD models, e.g., those of Horowitz et al. [12 14], study the possible nontrivial effect of introducing a second alternate position of sticking (see Fig $1 \mathrm{c}[\mathrm{d} / \mathrm{e})$. Each incoming particle either deposits on top of a selected site as in RD, or, sticks to a higher location of a taller nearest neighbour. The probability assigned is ad hoc and is not suggested by any underlying mechanism or dynamics. The GBD, on the other hand, represents a true stochastic process. The incoming particle, at each contact with the surface has a chance to stick (with probability $p$ ) or to slide down (with probability $q=1-p)$. This process continues for successive points of contact until the descending particle reaches the bottom of the column. The case of particle descending between two nearest neighbor adjacent columns is also considered, where the probabilities of sticking to two adjacent surfaces of contact is different than that for a single nearest neighbor column (see Fig. 1f).

In some other competitive growth models, different species of particles are considered, some deposit as in $\mathrm{RD}$, others as in BD. These models use mixture of particles with different pre-assigned probability of sticking. Thus, they represent deposition of mixed species of particles. However, no individual particle has the possibility of sticking to successive contact locations guided by a relevant stochastic process. Thus, in these other varaints of $\mathrm{BD}$ and competitive growth models, the possible sticking positions of the new particle are far fewer than those in the GBD model proposed in this work. The configurations possible in the competitive growth models (Fig 1c]1d]1e) form a smaller subset of the large ensemble of configurations allowed in the present model(Fig 1f).

The present GBD model involves stochasticity at two stages, first in the random selection of an active site and second, in the assignment of a sticking probability of the incoming particle. The structure of the deposit in the GBD model, depends on the successive stages of evolution. Hence, the relevant evolution equation is expected to be a stochastic integro-differential equation (SIDE) [10, 23]. This is in contrast to Edwards-Wilkinson (EW) or Kardar-Parisi-Zhang (KPZ) equations [5, 6] which are stochastic differential equation (SDEs).

\section{GENERALIZED BALLISTIC DEPOSITION MODEL}

The generalized ballistic deposition model presented in this work attempts to represent the deposition of realistic particles with varying degrees of stickiness. A parameter $p$, whose values vary between 0 and 1 , is introduced that represents the probability of an incoming particle sticking to a point of contact on the growing surface. A particle is allowed to descend vertically towards a randomly chosen

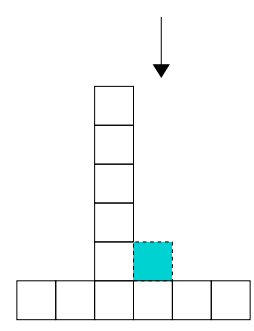

(a) Random deposition

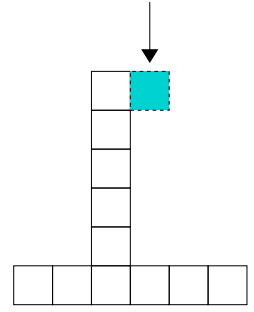

(b) Ballistic deposition

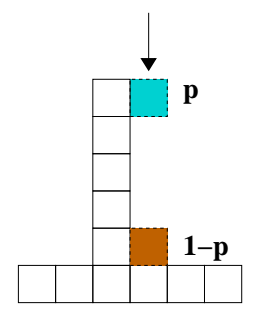

(c) HABD

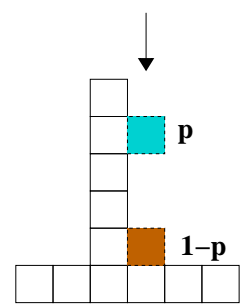

(d) HABD1

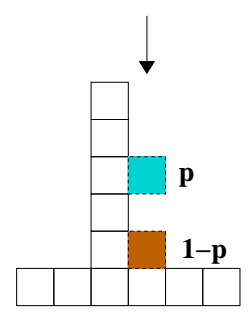

(e) HABD2

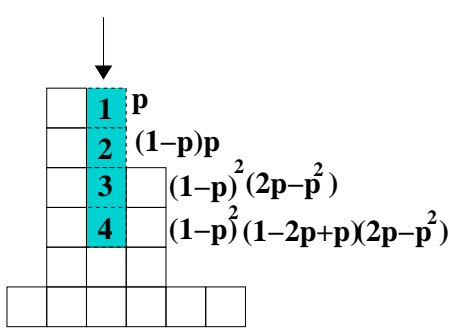

(f) present model (GBD)

FIG. 1: (Color Online) Allowed positions of the deposited particle for (a) RD, (b) BD,

(c, d, e) Horowitz and Albano's BD appearing in 13, 14, HABD, HABD1, HABD2,

(f) present model (GBD).

site on a one dimensional substrate. If the selected site is higher than its nearest neighbors, the particle simply deposits on top of the column at that site. However, if the chosen site has a taller column of particles as its nearest neighbor, then the new particle sticks to the first occupied site it encounters if the value of $p$ is larger than a random number generated from a uniform distribution between 0 and 1 . Otherwise, it slides down vertically to the next occupied site with probability $(1-p)$. At this site the particle may stick with probability $p(1-p)$ or continue its further descent with probability $(1-p)^{2}$, and so on, till it reaches the bottom. Thus if the chosen site has a nearest neighbor with column height taller by $n$ layers relative to it, the probabilities of the arriving particle sticking to the successive particles of the nearest neighbor column from top are given by,

$$
P(1)=p, P(2)=p(1-p), \ldots P(k)=p(1-p)^{(k-1)} .
$$

The probability that the particle slides past the preceding $(n-1)$ occupied neighbors, and lands at the lowest 
possible position is given by,

$$
P(n)=1-\sum_{k=1}^{n-1} P(k)=(1-p)^{(n-1)} .
$$

This describes a proper stochastic process. The total probability of a descending particle sticking to one of the allowed position is $\sum_{k=1}^{n} P(k)=1$.

In simple deposition models, the surface width follows a dynamic scaling law [24],

$$
W(L, t) \sim L^{\alpha} f\left(t / L^{z}\right),
$$

where $f$ is a scaling function satisfying $f(\infty) \sim$ constant and $f(x) \sim x^{\beta}$ for small $x$. The exponents $\alpha, \beta$ and $z$ are related by $z=\alpha / \beta$.

For the GBD studied in this work, the introduction of a sticking probability $p$, brings in another parameter in the problem. GBD interpolates between $\mathrm{RD}(p=0)$ and $\mathrm{BD}(p=1)$ systems. Physically relevant quantities, e.g., surface width $W(L, p, t)$ and porosity $\rho(L, p, t)$ thus depend on the sticking probability $p$ in addition to $L$ and $t$. From the results of our simulation we obtain dynamic scaling relations,

$$
\begin{gathered}
W(L, p, t) \sim L^{\alpha} p^{-\alpha^{\prime}} F\left(\frac{t p^{z^{\prime}}}{L^{z}}\right) \\
\rho(L, p, t) \sim L^{a} p^{b} G\left(\frac{t p^{d}}{L^{c}}\right) .
\end{gathered}
$$

where $F(x)$ and $G(y)$ are scaling functions described above.

\section{RESULTS AND DISCUSSION}

For different values of sticking probabilities between $p=0$ and $p=1$, simulations were performed in $(1+1)$ dimension for system sizes $L=16,32,64,128,256,512$ and 1024 . The graphs are suitably drawn to present the data, observations and results in a succinct, yet clear and uncluttered manner. The average number of layers deposited, is used as a measure of time $t$. Depending on the values of $p$ and $L$, the simulation results were averaged over 1000 to 5000 ensembles. The logarithmic plot of surface width with time has four distinct regions, for any non-zero probability $(p>0)$. This is shown in Fig 2 .

The dependence of surface width $W$ on $t$ in log-log scale, in the early submonolayer region $(t \ll 1)$ is linear with slope $1 / 2$ as in random deposition (growth region 1, GR-1). At later stages of submonolayer growth (growth region $2, \mathrm{GR}-2$ ), $t \simeq 1^{-}$, the surface width shows a steep increase which continues for the first few layers $(1-\epsilon \leq t \leq 3,1 \gg \epsilon>0)$. With deposition of further layers, the rate of increase in width slows down (growth region 3, GR-3). After deposition of a large number of layers, the ensemble average of the surface width saturates. Three different crossover times are of relevance. The first crossover time $t_{r}$ corresponds to the change from random growth to region with slope greater than $1 / 2$. The second crossover time $t_{k}$ corresponds to time beyond few layers where the slope decreases and changes from GR-2 to GR-3. The third crossover time $t_{\text {sat }}$ corresponds to beginning of saturation region.

The appearance of different growth regions in the present model may be understood as follows. Initially, when the deposition starts from a flat substrate almost no two adjacent sites are occupied. Hence there is no correlation among neighboring columns and the growth is random like, irrespective of whether the model allows for sticking or not. This feature is observed in all systems with different $L$ and $p$ values as shown in Fig 2 and Fig 3

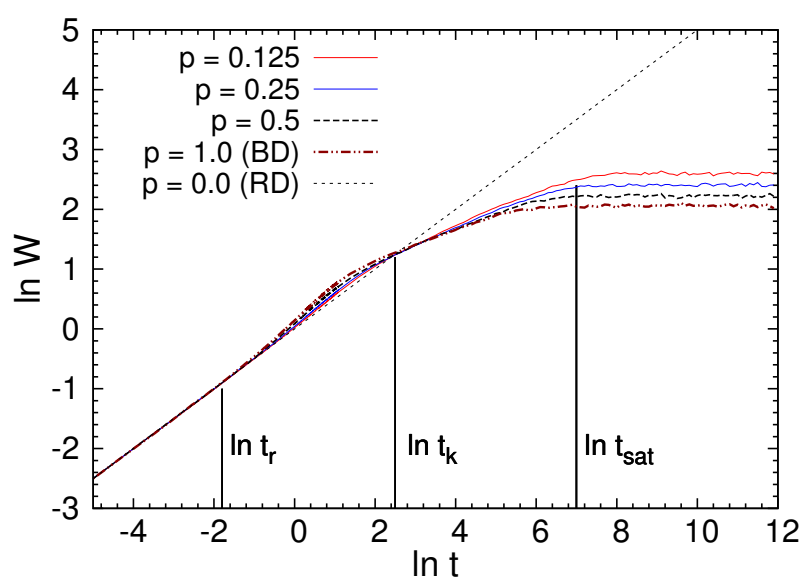

FIG. 2: (Color Online) Logarithmic plot of surface width with time for different $p$ and system size $L=256$, showing four distinct regions and crossover times.

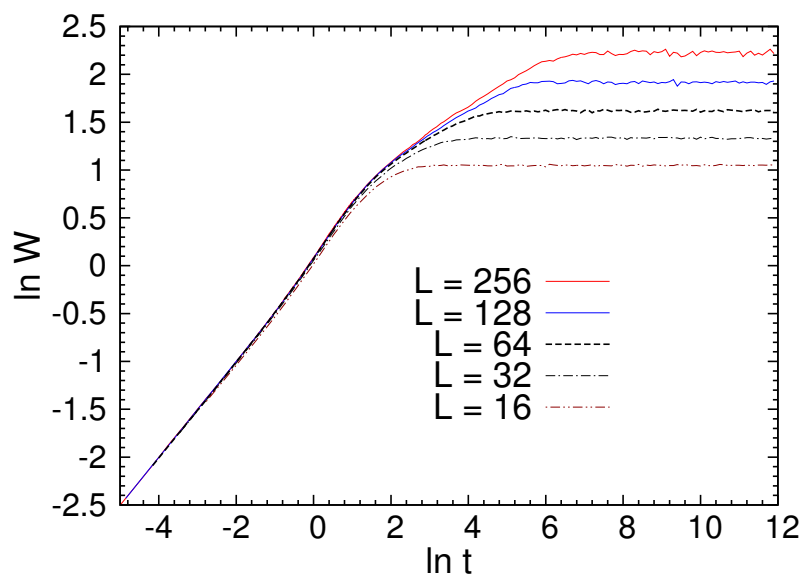

FIG. 3: (Color Online) Variation of $\ln W$ with $\ln t$ for $p=0.5$ for different system sizes. 
The deviation from random like behavior in the few layer deposition region is shown in Fig 4 . The rate of growth of surface width in this region is higher than that in the case of random deposition. As the number of particles deposited is nearly $L$, due to fluctuation, some short multi-layer columns may start forming. Hence the descending particles encounter occupied neighbors, and allowing sticking in the model, brings in non-trivial correlations in the system. However, since very few layers are deposited at this stage, even one particle sticking to a higher location or descending to the bottom of a column, makes a significant relative change in width. Thus the rate of growth of surface width in this region, when only a few layers have formed, is higher than that for RD. Our study shows, that the growth exponent in this region, denoted by $\beta^{\prime}$, increases with sticking probability $p$, as is shown in Fig 5, reaching a maximum when $p=1.0$, corresponding to the standard $\mathrm{BD}$, and is almost independent of system size $L$, as is shown in Fig 3 .

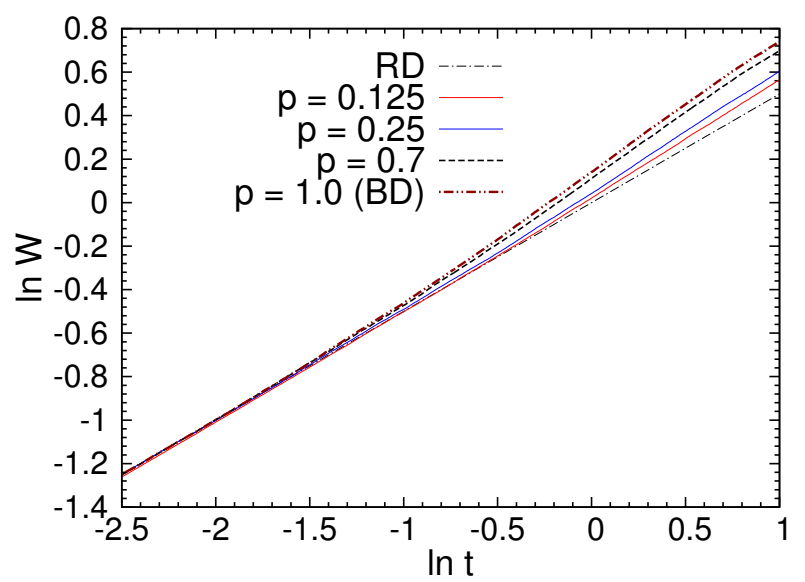

FIG. 4: (Color Online) Deviation from random deposition behavior at later stages of submonolayer growth for $L=256$.

The rate of increase in surface width slows down as more and more particles are deposited in GR-3. The average height of the interface and its width are larger. The descending particle need not proceed to the bottom, and can get deposited at a higher location by sticking. Thus the correlation has a smoothening effect, as it fills up deep crevices efficiently. The growth exponent $\beta$ in this region decreases with $p$, unlike the exponent in GR2. With further deposition of particles the surface width finally saturates. The saturated width depends both on the system size $L$ and sticking probability $p$, as shown in Fig 2 and Fig 3 .

For a given value of $p$, the surface width at saturation $W_{\text {sat }}$, and the time at which the saturation is reached $t_{\text {sat }}$, increase with system size $L$. For a given system size $L, W_{\text {sat }}$ and $t_{\text {sat }}$ decrease with increase in probability of sticking $p$. This decrease is more pronounced for lower values of $p$.

The dependence of $W_{\text {sat }}$ on $p$, for a given system size, is

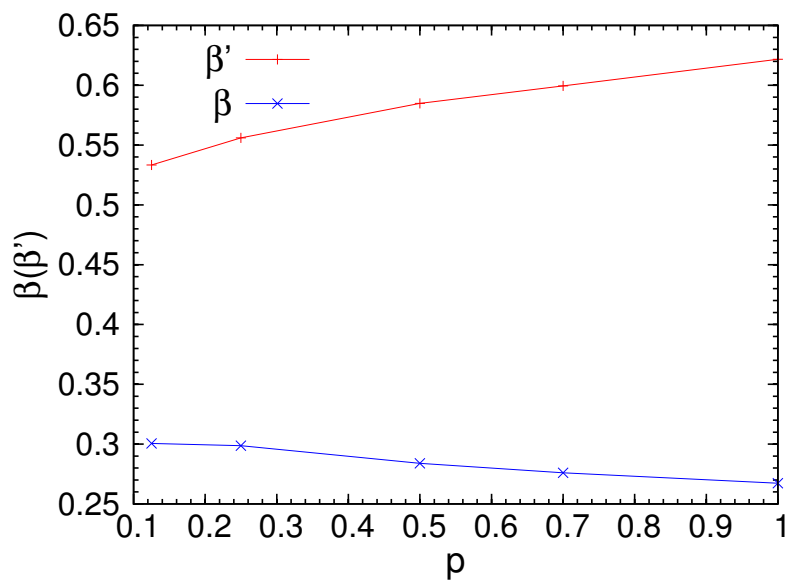

FIG. 5: (Color Online) Dependence of $\beta^{\prime}$ and $\beta$ on $p$ shown for system size $L=512$.

of the form $W_{\text {sat }}(L, p) \simeq f(L) \cdot p^{-\alpha^{\prime}}$, where the exponent $\alpha^{\prime}$ is independent of $L$ (Fig 6 ). An increase in sticking probability $p$ corresponds to a stronger correlation in the deposition process. The surface width saturates at lower values of saturation width $W_{\text {sat }}$, at corresponding earlier times, i.e., smaller $t_{\text {sat }}$.

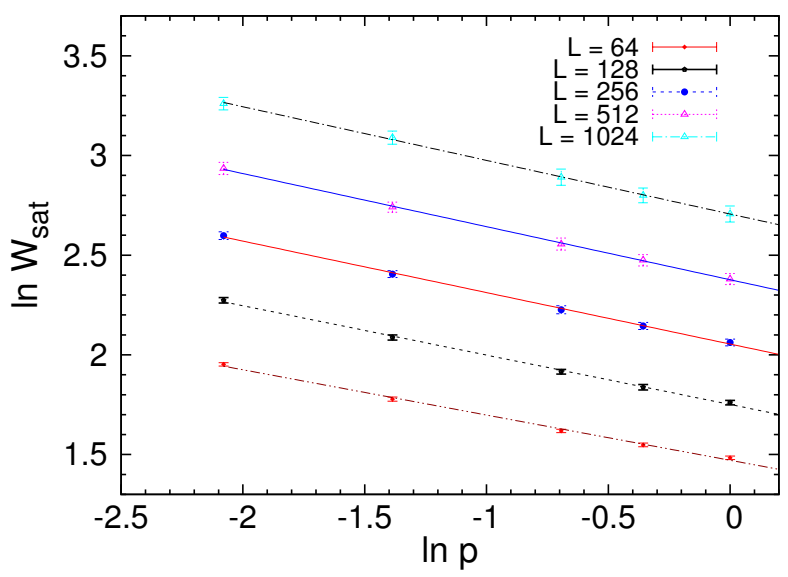

FIG. 6: (Color Online) Variation of $\ln W_{\text {sat }}$ with $\ln p$ for different system sizes $L$.

For a given probability of sticking $p, \ln W_{\text {sat }}$ increases linearly with $\ln L$. The dependence is found to be of the form $W_{\text {sat }}(L, p) \sim L^{\alpha}$ (Fig 7). From results of extensive simulations, graphically presented in the adjacent figures, Fig 6, Fig 7] and Fig 8, we find the exponents $\alpha=0.452$ and $\alpha^{\prime}=0.250$, in the region of saturated surface width.

The surface width $W$ in the third growth region, GR3 , i.e., between $t_{k}$ and $t_{s a t}$ depends on both system size $L$ and sticking probability $p$ as shown in Fig 9. The scaled width $\left(W p^{\alpha^{\prime}} / L^{\alpha}\right)$ in GR-3 shows that it is larger for larger system sizes and lower probability $(p=0.125)$. For a given system size $L, t_{\text {sat }}$ and hence the growth region, decrease with increase in probability $p$. The scaling 


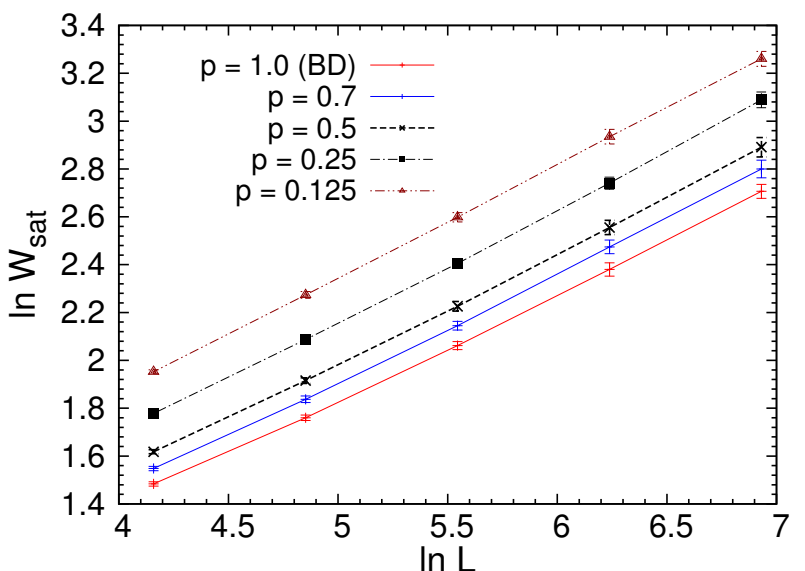

FIG. 7: (Color Online) Variation of the saturated surface width $\ln W_{\text {sat }}$ with $\ln L$ for different $p$ values.

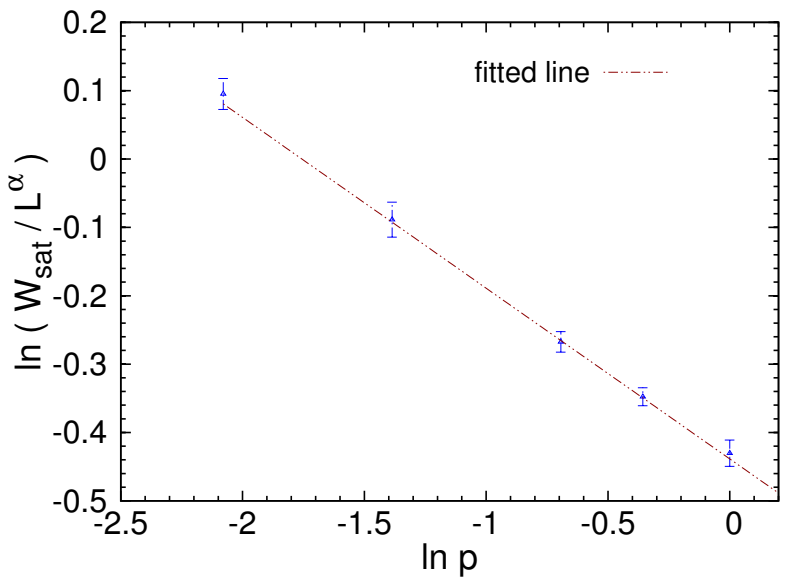

FIG. 8: (Color Online) Scaled saturated surface width $\ln \left(W_{\text {sat }} / L^{\alpha}\right)$ versus $\ln p$. The slope is -0.250 .

of $t$ with respect to $p$ is obtained from Fig 9, with exponent $z^{\prime}=0.7722$. Log-log plot of rescaled variables $\left(W p^{\alpha^{\prime}} / L^{\alpha}\right)$ versus $\left(t p^{z^{\prime}} / L^{z}\right)$ shows an excellent collapse of data in the growth region GR-3 and saturation region as shown in Fig 10 and Fig 11]

Analysis of our simulation data produces numerical estimates for the scaling exponents in Eq, The exponents are, $\alpha=0.452 \pm 0.016, \alpha^{\prime}=0.250 \pm 0.016$, $z=1.45 \pm 0.22$ and $z^{\prime}=0.77 \pm 0.04$. The closest representation of these values in terms of rational fractions are $\alpha=1 / 2, \alpha^{\prime}=1 / 4, z=3 / 2$ and $z^{\prime}=3 / 4$. From the scaled $W$ versus scaled $t$ graph in Fig 10 and Fig 11 , we obtain $\beta \simeq .312$ as the slope in the growth region GR-3. In Eq. 4, for small values of $\left(t p^{z^{\prime}} / L^{z}\right)$, we can approximate $F(x) \simeq x^{\beta}$, and hence

$$
W \sim L^{\alpha} p^{-\alpha^{\prime}}\left(\frac{t p^{z^{\prime}}}{L^{z}}\right)^{\beta},
$$

giving $\beta$ as the exponent of $t$ in this regime. The expo-

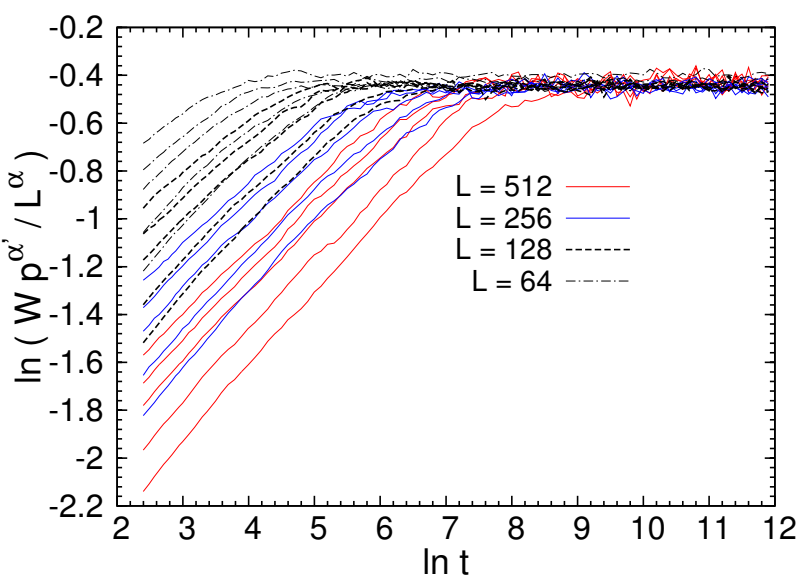

FIG. 9: (Color Online) Variation of $\ln \left(W p^{\alpha^{\prime}} / L^{\alpha}\right)$ with $\ln t$ showing dependence on system size $L$ and sticking probability $p$. Within each set of $L$, the different $p$ lines appear in order of decreasing $p$ values as one moves from bottom to top of the plot.

nents obtained above, satisfy the relations,

$$
\beta=\frac{\alpha}{z}=\frac{\alpha^{\prime}}{z^{\prime}}
$$

The growth exponent $\beta$ is approximately the rational fraction $1 / 3$.

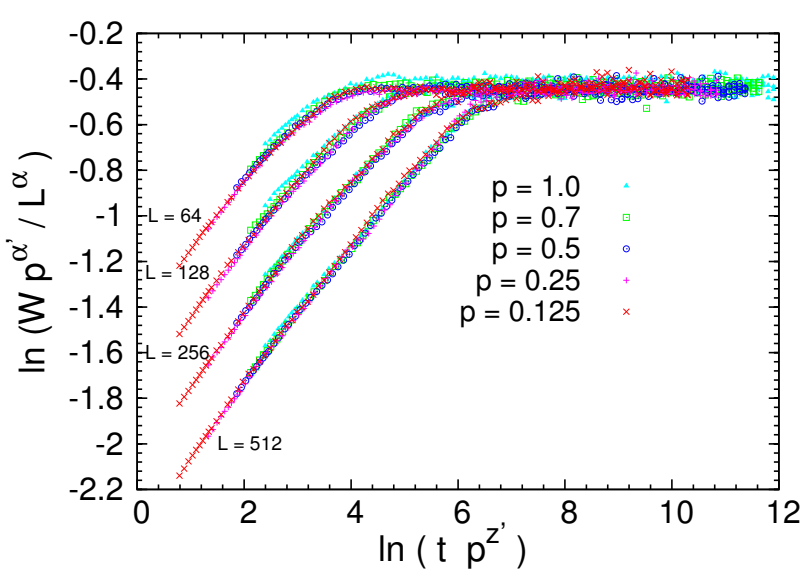

FIG. 10: (Color Online) $\ln \left(W p^{\alpha^{\prime}} / L^{\alpha}\right)$ versus $\ln \left(t p^{z^{\prime}}\right)$ for different sticking probability $p$.

The porosity $\rho$ can be defined as the fraction of unoccupied sites within a few layers $N$ just below the active interface, where no further deposition can take place. It is seen that the porosity is quite independent when $N$ is varied from 16 to $L$ for a given system size $L$. The porosity is found to initially increase with time, signifying growth and then saturates to a value $\rho_{\text {sat }}$. The onset of saturation for porosity occurs earlier than the onset of saturation of surface width.

The porosity $\rho$ is found to depend on the sticking probability $p$ and shows a weak dependence on the system size 


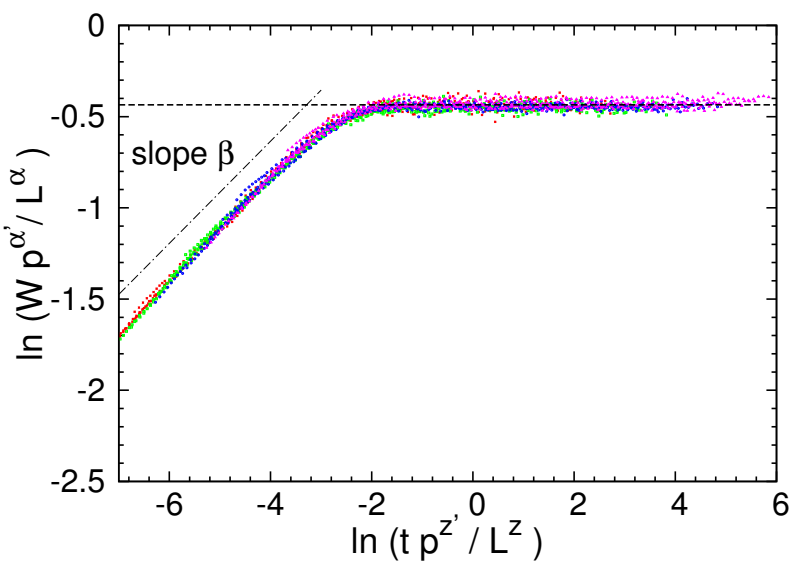

FIG. 11: (Color Online) $\ln \left(W p^{\alpha^{\prime}} / L^{\alpha}\right)$ versus $\ln \left(t p^{z^{\prime}} / L^{z}\right)$ for $L=512,256,128,64$ and $p=1.0,0.7,0.5,0.25,0.125$.

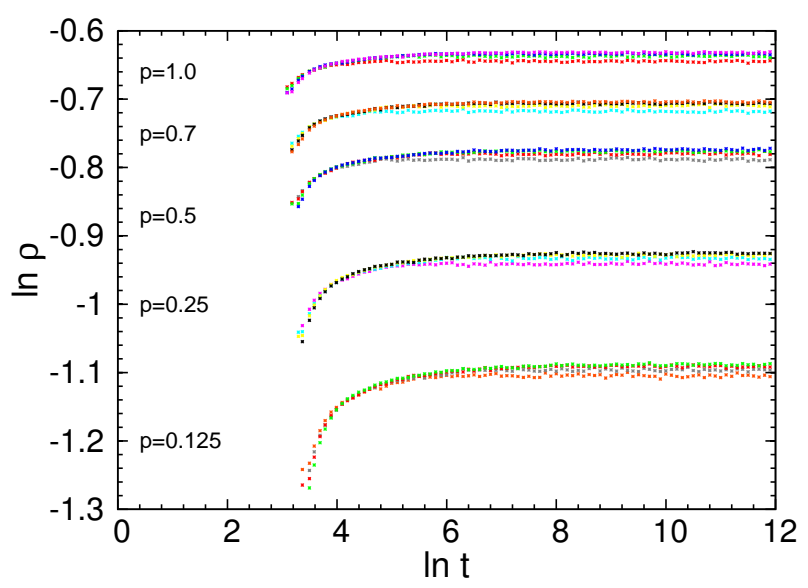

FIG. 12: (Color Online) $\ln \rho$ versus $\ln t$ for $L=512,256,128,64$ and $p=1.0,0.7,0.5,0.25,0.125$.

L. $\rho_{\text {sat }}$ is higher for higher $L$ at any given $p$. However, in the early growth region, $\rho(L, p, t)$ starts at a lower value for higher $L$. The porosity in both the growth and saturation regions increases with increase in sticking probability $p$; fully ballistic deposition system being most porous. Onset of saturation in porosity is earlier for higher sticking probability. A scaling behavior for porosity is obtained in the form of Eq5, with the exponents $a=0.00690 \pm 0.00070, b=0.2204 \pm 0.0051, c=$ $0.134 \pm 0.035$ and $d=0.59 \pm 0.16$. The dependence of porosity on time $t$, sticking probability $p$ and system size $L$ is shown in Fig 12 and as scaled data in Fig 13 .

It may be noted that, in our simulations of the present generalized ballistic deposition model, two independent random number generators were used, one for selecting a site on the growing surface and, another to determine whether a particle will stick at a particular location for a chosen value of the sticking probability. These two random number generators are completely independent

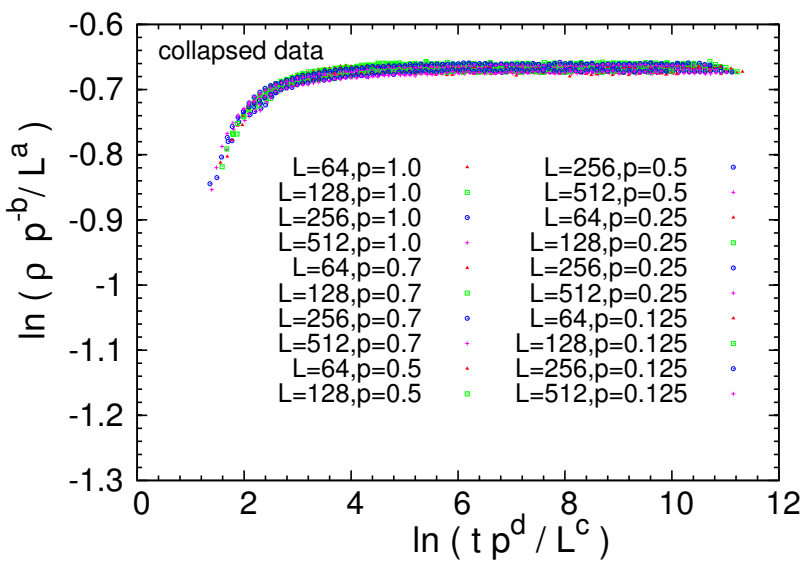

FIG. 13: (Color Online) Scaled data shown as $\ln \left(\rho p^{-b} / L^{a}\right)$ versus $\ln \left(t p^{d} / L^{c}\right)$ for $L=512,256,128,64$ and $p=1.0,0.7,0.5,0.25,0.125$.

and uncorrelated to each other.

\section{CONCLUSION}

We have studied the deposition of physically realistic particles with variable stickiness by proposing a generalized ballistic model of deposition.

As mentioned in section II] the GBD model involves stochasticity in two stages:(i) in random selection of an active site and (ii) in assigning a sticking probability that affects the extent to which the height at the active site is altered for a given configuration of particles deposited on neighboring sites. The random selection of an active site appears as the random noise term in the corresponding differential equation, such as in the KPZ equation, while the detailed mechanism of the change in height $h(x, t)$, determines the different spatial derivatives i.e., slope, curvature etc., appearing in the differential equations.

In the present model, the sticking of an incoming particle at a site is possible only when it has slipped past the surfaces encountered earlier in its path. Thus, the possibility of sticking at the present position, depends on the probabilities of all such earlier events. The relevant equation and the scaling behavior is expected to depend on this sticking probability. This is corroborated by our observations.

A larger value of sticking probability implies stronger correlation among neighboring columns, hence, the surface width, at a fixed value of $L$, is expected to saturate to lower values of $W_{\text {sat }}$ at earlier (smaller $t_{\text {sat }}$ ) times. In the growth region, however, correlations have a smoothening effect, as deep creivces are filled up more efficiently. Thus, in the growth region, surface width, at a fixed $L$, should decrease with increase in sticking probability. Porosity, on the other hand, should increase with the sticking probability, in both growth and saturation regions for a fixed 
system size, as a larger sticking probability implies a more porous structure. Our observations are in agreement with the above arguments.

We find excellent collapse of data for the scaling of the surface width and porosity, in terms of system size $L$ and sticking probability $p$ in the KPZ-like growth and saturation regions.

A simplying assumption in this work is that the sticking probability at successive encounters is assumed to be a constant. However, each encounter may cause some physical change(s) to the incoming particle. Further investigation of the above may be of interest and progress of study along the above lines will be reported elsewhere.
It may be noted however, that a most general treatment will involve all the complexities associated with non-Markovian processes.

\section{ACKNOWLEDGEMENT}

Authors gratefully acknowledge the use of computing facility at University of Calcutta, under the DRS program sponsored by the University Grants Commission, India. B. M. also wishes to acknowledge the financial assistance provided by the West Bengal State Departmental Fellowship, India.
[1] A. L. Barabasi and H. E. Stanley, Fractal Concepts in Surface Growth (Cambridge University Press, Cambridge, 1995).

[2] P. Meakin, Physics Reports 235, 189 (1993).

[3] S. Boncel, A. P. Herman, and K. Z. Walczak, J. Mater. Chem. 22, 31 (2012).

[4] K. M. Kummer, E. Taylor, and T. J. Webster, Nanosci. and Nanotechnol. Lett. 4, 483 (2012).

[5] S. F. Edwards and D. R. Wilkinson, Proc. R. Soc. Lond. A 381, 17 (1982).

[6] M. Kardar, G. Parisi, and Y.-C. Zhang, Phys. Rev. Lett. 56, 889 (1986)

[7] D. D. Vvedensky, Phys. Rev. E 67, 025102 (2003)

[8] F. Family and T. Vicsek, J. Phys. A: Math. Gen. 18, L75 (1985).

[9] F. Family, Physica A: Statistical Mechanics and its Applications 168, 561 (1990).

[10] K. Banerjee, J. Shamanna, and S. Ray, Phys. Rev. E 90 , $022111(2014)$.

[11] E. Katzav, S. F. Edwards, and M. Schwartz, Europhys. Lett. 75 (1), 29 (2006).

[12] C. M. Horowitz, R. A. Monetti, and E. V. Albano, Phys. Rev. E 63, 066132 (2001).
[13] C. M. Horowitz and E. V. Albano, J. Phys. A: Math. Gen. 34, 357 (2001).

[14] C. M. Horowitz and E. V. Albano, Phys. Rev. E 73, 031111 (2006)

[15] W. Wang and H. A. Cerdeira, Phys. Rev. E 47, 3357 (1993).

[16] W. Wang and H. A. Cerdeira, Phys. Rev. E 52, 6308 (1995).

[17] H. F. El-Nashar and H. A. Cerdeira, Phys. Rev. E 61, 6149 (2000).

[18] F. D. A. Aarao Reis, Phys. Rev. E 66, 027101 (2002).

[19] F. D. A. Aarao Reis, Phys. Rev. E 68, 041602 (2003).

[20] F. D. A. Aarao Reis, Phys. Rev. E 73, 021605 (2006).

[21] Y. P. Pellegrini and R. Jullien, Phys. Rev. Lett. 64, 1745 (1990).

[22] Y. P. Pellegrini and R. Jullien, Phys. Rev. A 43, 920 (1991).

[23] E. Katzav, Phys. Rev. E 68, 046113 (2003).

[24] F. Family, J. Phys. A: Math. Gen. 19, L441 (1986). 